\title{
LEYENDO EL CONSUMO DESDE LAS EMOCIONES SOCIALES. Algunos recorridos y perspectivas posibles
}

\author{
Andrea DETTANO \\ Universidad de Buenos Aires (Argentina) \\ andreadettano@gmail.com
}

READING THE CONSUMPTION FROM THE SOCIAL EMOTIONS. Some possible perspectives

Resumen: Las prácticas de consumo han estado históricamente asociadas con las emociones, siendo dadoras de status, orgullo, prestigio, satisfacción, vergüenza. En este sentido, el presente escrito tiene por objetivo rastrear en algunos trabajos teóricos, el modo en que el consumo -como práctica social- ha estado fuertemente relacionado con las emociones de los sujetos. La estrategia expositiva será la siguiente: en primer lugar se hace un recorrido teórico que escinde el consumo de las necesidades vitales y de la noción de utilidad. En segundo lugar se consideran los trabajos de Erich Fromm (2013) y de Colin Campbell (1983), en cuyos desarrollos las emociones presentan un lugar central en vinculación con procesos económicos y sociales. En tercer lugar -acercándonos a una perspectiva más actual- , se buscará delinear los rasgos del "hombre de consumo del siglo XXI" (Scribano, 2015). Por último, se harán unas reflexiones a modo de cierre.

Abstract: Consumption practices have historically been associated with emotions, being givers of status, pride, prestige, satisfaction, shame. In this sense, the present paper aims to track in some theoretical works the way in which consumption -as a social practice- has been strongly related to the emotions of the subjects. The expository strategy will be the following: in the first place a theoretical route is made that clears the consumption of the vital necessities and the idea of utility. Secondly, we consider the works of Erich Fromm (2013) and the paper of Colin Campbell (1983), in whose developments the emotions present a central place in connection with economic and social processes. Thirdly, -approaching to a current perspective- we try to describe some aspects of "consumption man of the 21 st century" (Scribano, 2015). Finally, some reflections will be made as a closure.

Palabras clave: Consumo. Emociones. Prácticas. Estructura Social

Consumption. Emotions. Practices. Social Estructure 


\begin{abstract}
"Pero toda investigación que quiera entender la conciencia de los hombres, «su ratio» o sus «ideas» sin considerar al mismo tiempo la estructura de los impulsos, la orientación y la configuración de los sentimientos y de las pasiones solo conseguirá resultados limitados, puesto que ignorará necesariamente gran parte de lo que es imprescindible para los seres humanos" (Elías, 2009: 588).
\end{abstract}

\title{
Introducción
}

Las prácticas de consumo han estado históricamente asociadas con las emociones, siendo dadoras de status, orgullo, prestigio, satisfacción, vergüenza. En este sentido, el presente escrito tiene por objetivo rastrear en algunos trabajos teóricos el modo en que el consumo -como práctica social- ha estado fuertemente relacionado con las emociones de los sujetos.

Para los estudios sociales sobre las emociones, la configuración de estas, se vincula con la manera en que los sujetos viven y perciben el mundo, el impacto de la vida cotidiana como sucesiones de hechos y acontecimientos que van configurando las maneras ver, verse y relacionarse con los otros. El estudio de las emociones es abordado, como resultante de las prácticas de ser, hacer y habitar el mundo, por lo que su carácter es eminentemente social.

Preguntarse acerca de las emociones y su relación con prácticas de consumo, implica plantearse un modo de estructuración particular que habilita ciertas prácticas y restringe otras. Un ejemplo de esto podría ser lo que Weber describe en "La Ética protestante y el espíritu del capitalismo", en tanto el intento de agradar a Dios organizaba las acciones terrenales, lo que devino en el desenvolvimiento de procesos de acumulación económica.

Este trabajo se concentrará en indagar diversas perspectivas teóricas sobre el consumo, el modo en que adquirir objetos y el uso, se ha ido transformando en un hacer cotidiano, habitual y masivo, y el modo en que esto se relaciona con determinadas formas del sentir, como el placer, el disfrute, la resignación, la felicidad, la ilusión, entre otras.

La estrategia expositiva será la siguiente: en primer lugar se hace un recorrido teórico que escinde el consumo de las necesidades vitales. En segundo lugar se consideran los trabajos de Erich Fromm (2013) en "Ser o Tener" y de Colin Campbel (1983), en cuyos desarrollos las emociones presentan un lugar central en vinculación con procesos económicos y sociales. En tercer lugar se buscará profundizar la relación entre consumo y emociones hasta llegar a la descripción del "hombre de consumo del siglo XXI (Scribano, 2015). Por último, se harán unas reflexiones a modo de cierre.

\section{Consumo, utilidad y necesidades: algunas precisiones para comenzar}

En los entramados actuales, denominados como sociedades del capitalismo tardío (Jameson, 2002) o sociedades de consumo (Bauman, 2007; Baudrillard, 2010), los agentes realizan la producción y reproducción de sus vidas cotidianas atravesados por el consumo en tanto forma de ser y estar con otros (Dettano y Lava, 2014). Mientras que a nivel individual esta práctica es vivenciada como un conjunto de decisiones propias, íntimas, particulares, podemos sostener que, en tanto práctica social, "la actividad del consumo es la producción colectiva, con sus respectivos consumidores, de un universo de valores" (Douglas, Isherwood, 1990: 83), de forma que no hay consumidor ni bienes aislados.

Las teorías de consumo basadas en el comportamiento racional del consumidor, cuya expresión fue el homo oeconomicus, tienen una raigambre marginal situada a finales del siglo XIX y da por entendido que las características de un bien podían satisfacer las necesidades humanas. Para dicha perspectiva, los "hechos económicos" se limitaban al espacio del mercado donde en una situación de competencia perfecta se formaban los precios y los sujetos solo deberían elegir en base a utilidad y esquema de precios más conveniente en pos del logro de una mayor satisfacción. En este sentido: "La sociedad queda conformada así, como un conglomerado de individuos soberanos, no sometidos a ninguna relación como ser social; particularmente, el consumo, no tendrá más vínculo con el resto de los procesos económicos y sociales que el mercado y la formación de precios" (Alonso, 2005: 4). Así, el consumo no sería más que un acto, producto de un cálculo racional que atañe al mundo privado del sujeto basado en unas preferencias que operan con anterioridad a intercambios y relaciones sociales.

Tomando cierta distancia con respecto a estas posiciones, otros autores han ido elaborando diferentes perspectivas que vinculan la práctica en cuestión a las formas de vivir mutuamente implicados de los su- 
jetos, es decir, lo social. En primer lugar, para Marx, la "utilidad" el modo en que una mercancía se usa, satisface necesidades, tiene una constitución histórica. En su amplio desarrollo teórico sobre la mercancía, nos dirá que esta, satisface necesidades humanas de cualquier tipo y que "la naturaleza de esas necesidades, el que se originen, por ejemplo, en el estómago o en la fantasía, en nada modifica el problema" (2008: 43).

Veblen aportará en la misma dirección al hablar de "consumo pecuniario" y "emulación". Así, los patrones de consumo para Veblen son hábitos, que poco tienen que ver con la optimización del gasto, o con la noción de utilidad. Es central lo que define como "instituciones" en tanto "Hábitos mentales predominantes con respecto a relaciones y funciones particulares del individuo y de la comunidad" (Veblen, 1944: 196). Dichas instituciones, son una guía para el comportamiento individual, lo afectan y podemos agregar, no surgen del individuo particular. Esas guías para el comportamiento no se basan en un principio optimizador, como el que describe la economía clásica, sino que se asientan en hábitos.

En esta línea, la noción de gasto desarrollada por Bataille (1933) nos permite tener en cuenta que no hay modo de definir lo que es útil a los hombres, o al menos aquello que desde la perspectiva de los científicos sociales y la economía, consideramos como tal. La pérdida y el derroche en instituciones como el Potlatch exponen que la productividad, acumulación y utilidad no es lo que necesaria o esencialmente explica el sentido de la acción. De hecho, Bataille, alejándose de aquella economía restringida, otorga a la perdida y al derroche una propiedad positiva, asociada al honor, la nobleza, las jerarquías, constituyendo lo que denomina "una economía general" (Fava, 2009).

Entonces, no es el resultado de un cálculo racional, no responde a necesidades meramente biológicas, pero tampoco se reduce al nivel de ingresos (Lipovetsky, 2007). La práctica de consumo no se vincula únicamente con el nivel de ingresos, o con la utilidad, sino que se encuentra atravesada por sentidos y emociones sociales que resultan en guías para la acción.

En este sentido, para Bauman (2007) hablar de consumo no restringe al terreno de las necesidades vitales ni de la reproducción biológica de los agentes, sino que se vincula más con aquello que define como "consumismo", que pareciera estar fuertemente vinculado con una específica forma de sentir. Allí, la práctica de adquisición de objetos se vuelve central, exacerbando "nuestra capacidad de querer, de desear y de anhelar, y en especial de experimentar esas emociones repetidamente como el fundamento de toda la economía de las relaciones humanas" (Bauman, 2007: 44).

La relación entre sujetos, consumo y emociones, como veremos a continuación, se vincula con la búsqueda del disfrute, la resignación, con transformaciones en las concepciones sobre el placer, en el lugar del yo o self. Hablar de las emociones en la actualidad ligadas al consumo y al disfrute inmediato (Scribano, 2015) -como veremos a lo largo del escrito- conlleva dar cuenta no solo de una orientación a la adquisición constante, uso, o de persecución del goce, sino que implica considerar las formas crecientes de mercantilización de la vida (Hochschild, 2011) y de una serie de mecanismos que tienden a la evitación del conflicto.

\section{Consumo, emociones y sociabilidades}

En este apartado recuperaremos aspectos de dos trabajos que colaboran en la reflexión acerca de la relación entre consumo y emociones sociales. Como punto de partida, diremos que los seres humanos solo podemos experimentar la vida emocionalmente (Bericat Alastuey, 2012) y que estas emociones han sido parte constitutiva de la realidad histórica, plagada de sujetos sentientes que formaron parte de procesos, devenires y transformaciones. Si bien parecen ser lo más íntimo de cada sujeto, algo meramente individual, podemos sostener que, "Las emociones humanas, al igual que el lenguaje, están constituidas por procesos en los que se cruzan factores aprendidos y no-aprendidos. Esta hipótesis significa que, al constituirnos socialmente como adultos funcionales, hemos vivido un proceso de aprendizaje que ha modelado los componentes emocionales no-aprendidos en la clave social del grupo al cual pertenecemos" (Sánchez Aguirre, 2013: 79).

Las emociones han estado muy presentes en las teorías sociológica clásicas, sin embargo, los estudios sociales sobre las emociones -como campo dentro de la disciplina- nacen en la década de los años 70 del siglo XX, de la mano de algunos pioneros norteamericanos como Arlie Russell Hochschild, Thomas Scheff, Randall Collins y Theodore Kemper, quienes hacen las primeras publicaciones vinculadas con la temática. Su estudio implica considerarlas como producto del intercambio de los sujetos entre sí y con el ambiente, como el resultado de habitar el mundo (Scribano, 2009). En ese intercambio se van configurando regulaciones emocionales que resultan en guías para la acción (Elías, 2009). De esta forma, si consideramos que "la emoción es necesaria para explicar los fundamentos de la conducta social" (Bericat Alastuey, 2012: 4), entonces, se buscará rastrear el modo en que éstas así como variadas formas del sentir están presentes en los trabajos a considerar y como se han vinculado estrechamente a la proliferación de prácticas de consumo.

Una pregunta que sería pertinente responder, es porque se han elegido estos textos y no otros para pensar la relación entre el consumo y las emociones en tanto objeto de indagación social. La elección tuvo que ver con el modo en que los mismos consideran en sus análisis formas de sentir vinculadas con las prácticas de 
consumo. En el escrito de Campbell hay una fuerte intención en no ver el consumo de un modo negativo, en tanto práctica propia de un sujeto hedonista. Por el contrario, en la obra de Fromm puede leerse una mirada en donde el consumo impacta en la subjetividad de modo que el yo queda orientado hacia el "tener" -portador de ciertas connotaciones negativas- en contraposición a un "ser", dotado de atributos positivos. La riqueza de los textos utilizados es que ambos exhiben la complejidad de la ligazón entre una práctica determinada y una forma particular de sentir en el mundo.

El primer escrito a considerar es "Ser o Tener" (2013), de Erich Fromm. Este trabajo tanto como el de Colin Campbell intenta describir cambios en los sujetos que resultan afines a la proliferación de prácticas de consumo. Fromm comenzará delineando algunos aspectos del paso a la modernidad. Dicho "paso" acarreó una serie de transformaciones. En primer lugar, en el medioevo la conducta económica estaba determinada por principios éticos, mientras que en el siglo XVIII atravesó un cambio producido por la separación entre la conducta económica y los valores humanos. El concepto de "ganancia" se escinde de una semántica vinculada a lo religioso, a la "ganancia del alma" y empieza a asociarse con una vinculada a lo material, a las transacciones mercantiles. La maquinaria económica fue logrando autonomía, regida por sus propias leyes, lo que devino en la aceptación de sus consecuencias como parte de un funcionamiento natural. Las iniciativas o acciones que eran beneficiosas para el sistema o un conjunto de empresas eran presentadas como la expresión del bienestar general. El siglo XVIII y sus cambios producto de las escisiones mencionadas modificaron para el autor la orientación del carácter humano, es por eso que el libro en cuestión se dedica a desentrañar dos modos básicos de la existencia como son el ser y el tener.

Situando las fuentes de la modificación del carácter humano en el siglo XVIII, Fromm describe cómo el desenvolvimiento económico producto de la industrialización, fue alimentando una esperanza relacionada con el posible bienestar que podría alcanzarse para la sociedad toda. Realizando su análisis desde el siglo XX, da cuenta del fin de la promesa del bienestar y la felicidad sin límites, prometido por la industrialización, y en segundo lugar, de una forma de la subjetividad -hedonismo radical o egoísmo-, que se desarrolla al calor de este aumento en la circulación de bienes y cambios en los modos de producirlos.

El "fin de una ilusión" es presentado como la caída de todas las esperanzas de progreso, abundancia y felicidad que albergaban los procesos de industrialización. El uso de la técnica nos haría omnipotentes, dice el autor, haciendo posibles la producción y el consumo ilimitados. El hombre sería capaz de utilizar la naturaleza para crear su propio mundo, para transformarlo de modo radical. Hombres y mujeres creían haber logrado la libertad, tras romper con las formas feudales de organización social, a la par que la fe en el progreso haría extensiva esta "libertad" a todos los sectores de la sociedad. Metas colectivas como la socialista y la comunista trocaron en la consecución de una "burguesía universalizada". En esta línea, "[1]a trinidad «producción ilimitada, libertad absoluta y felicidad sin restricciones» formaba el núcleo de una nueva religión: el Progreso y una nueva ciudad terrenal del Progreso reemplazaría a la Ciudad de Dios. No es extraño que esta nueva religión infundiera energías, vitalidad y esperanzas a sus creyentes" (Fromm, 2013: 21).

Esta nueva religión, redefine los elementos actuantes, las formas de estructuración del disfrute. Desde el siglo XVII, la cuestión del placer, ya había sido enarbolada por distintos filósofos, quienes abordaron la persecución constante de satisfacción, el deseo de placer como algo que por sí mismo merece ser atendido, y que fue ubicado en el cuerpo, en las sensaciones corporales. Lo que podía producir felicidad y placer fue ganando concesiones, espacios y permisos, adquiriendo una legitimidad tal que hechos antes severamente cuestionados -como mantenerse codicioso, consumir drogas buscando una ilusión de bienestar, e incluso la satisfacción de impulsos crueles- fueron proclamados por distintos pensadores y aceptados socialmente. Esto también se verá en los desarrollos de Campbell (1983).

Sin embargo, la búsqueda del placer o el placer constante no resuelven, para Fromm, la problemática de la existencia humana. En primer lugar la premisa del "hedonismo radical", donde una persona persigue obtener todo aquello que desea o produce satisfacción, resulta problemática, en términos de que la satisfacción ilimitada no conduce al bienestar o a la felicidad. En segundo lugar, también declina la premisa de que la satisfacción del egoísmo humano produce paz y armonía social. Estos factores generan una situación contraria, basada en el egoísmo como una disposición del carácter, que implica la acumulación individual, la avaricia, la competencia, la envidia hacia los otros. Esto lo lleva a definir dos orientaciones básicas del carácter humano, la del "tener" y las del "ser" como radicalmente opuestas.

La orientación del "tener", en la obra de Fromm, es característica de la sociedad industrial de occidente, donde el poder, el lucro y la fama son el problema o asunto dominante. Es por esto que el hombre moderno se rige por los principios de la propiedad y la codicia. Fromm exhibe como este atravesamiento de la lógica de la propiedad en la conducta humana puede visualizarse, incluso, en el uso del lenguaje. Su fundamentación reside en que la manera de hablar revela una alienación oculta, inconsciente. Si bien parece muy natural utilizar el vocablo "tener", e incluso enunciar la propiedad de objetos, hay idiomas que no poseen esa palabra, como en el hebreo, donde se usa una expresión indirecta para indicar posesión. La tesis del 
autor acerca del desenvolvimiento de la propiedad privada y el uso del lenguaje exhibe lo que define como "la orientación profunda del carácter humano" hacia la posesión de objetos y el nexo entre modos de producción, circulación de bienes y emocionalidades. En este sentido:

"Con ser o tener no me refiero a ciertas cualidades o propiedades de un sujeto en afirmaciones como estas: «tengo un auto», «soy blanco» 0 «soy feliz». Me refiero a dos modos fundamentales de existencia, a dos tipos distintos de orientación ante el yo y ante el mundo, a dos tipos distintos de estructura del carácter cuyo predominio respectivo determina la totalidad del pensamiento, de los sentimientos y de los actos de la persona" (Fromm, 2013: 40).

En cuanto al "ser", Fromm recupera y adopta la visión del ser de Heráclito y de Hegel, vinculada con el movimiento, la actividad, lo procesual. Desde esta concepción del ser no hay lugar para el concepto de sustancia, todo es proceso y devenir. El cambio y el desarrollo son cualidades inherentes al ser en tanto proceso vital. Para demostrar en que consiste se dan algunos ejemplos vinculados con el amor, la fe, el conocimiento, entre otros. En cada uno de estos aspectos, existe una forma de vivir de acuerdo al ser u otra de acuerdo al tener. Todo aquello vinculado al ser tiene que ver con una praxis, con actos y no con una apropiación. En cuanto al ejemplo del amor, este es visto como un acto, que implica cuidar, conocer, afirmar, gozar de alguien, aumentar su vitalidad, mientras que en el modo de "tener", implica aprisionar, encerrar, poseer o dominar al objeto amado.

Tener, se asocia a "incorporar", lo que consiste en una forma primaria de posesión. El niño lo hace, llevándose a la boca lo que desea. El canibalismo, por ejemplo, también se desenvuelve de esta forma, asociando la incorporación con la posesión, de modo que cuando incorpora a otro, o a "algo", asume sus características. Claro que también existe una forma de voracidad simbólica, que hace que el objeto forme parte del sujeto que lo "incorpora", poseyéndolo. De este modo, "La actitud inherente al consumismo es devorar todo el mundo. El consumidor es el eterno niño de pecho que llora reclamando su biberón" (Fromm, 2013: 43). Consumir es la forma primordial del tener en las sociedades industriales, lo que para el autor conlleva un efecto reductor de la angustia pero solo de forma momentánea.

Además de tomar los aportes de Fromm, consideraremos un artículo de Colin Campbell publicado en 1983 que aborda la relación entre una ética de consumo y el romanticismo como movimiento artístico y cultural, cuyo comienzo podría situarse a fines del siglo XVIII. Este trabajo realiza un análisis en clave Weberiana, observando o tratando de construir un mapa tanto del consumismo moderno como del romanticismo para ver la afinidad electiva que puede haber entre ambos fenómenos sociales.

Campbell (1983) invita, a su vez, a desmitificar la idea de que la sociedad de consumo parte de la segunda posguerra, sosteniendo que esta, es una característica del desarrollo de la producción industrial y del capitalismo. Por eso, intenta rastrear las bases del espíritu o el ethos del consumidor moderno y burgués en otros elementos más allá de las transformaciones en los modos de producir bienes.

La revolución que fue consolidando al proletariado urbano produjo al mismo tiempo al consumidor moderno. Campbell hace una línea demarcatoria entre el consumidor tradicional y el moderno. En la sociedad tradicional las ideas que enmarcan el consumo podrían ser bienes limitados y economía estática, "lo que se quiere" es una necesidad y está limitado. En la sociedad tradicional, dice Campbell, se maneja una economía de subsistencia para la mayoría de la población y las ideas acerca de esforzarse para estar mejor uno mismo son consideradas como algo cercano a la inmoralidad.

El asunto reside en una "imagen del hombre" propia del mundo cristiano feudal muy distinta a la del mundo burgués. En la sociedad feudal las personas primero son miembros de un cuerpo social y luego individuos. Para el cristianismo el cuerpo social y la comunidad existen por encima del individuo. El anonimato de los monjes en los monasterios es un ejemplo de como el sujeto ocupa un lugar insignificante. La imagen del hombre propia del mundo burgués hará una inversión, poniendo en primer lugar al individuo, en tanto empieza a considerarse como un universo completo en sí mismo (Romero, 2006).

La economía actual, tiene un funcionamiento diferente en cuanto al comportamiento económico de los sujetos, la producción creciente y los bienes ilimitados son considerados normales. En esta "normalidad", Campbell (1983), hablará de una revolución de las expectativas, que implica que no solo cada uno persigue el bienestar, el estar mejor sino que si no lo hace eso es considerado lo "inmoral". Hay que buscar nuevos deseos y perseguir satisfacerlos. "la obligación de satisfacer deseos está ligada a una economía de mercado corporeizando el principio del consumidor soberano así como garantizando mecanismos de perpetua estimulación de nuevos deseos" (Campbell, 1983: 281. Traducción propia).

Mientras que en la sociedad tradicional lo erótico, el amor, así como el beneficio o interés que se obtiene del dinero eran la imagen de lo pecaminoso, con el desarrollo de la burguesía comienzan a consolidarse como lo deseable y esperado, reflejando una nueva actitud moral (Romero, 2006). 
Ahora bien, lo que hubo tanto en la sociedad tradicional como en la sociedad moderna son guías de conducta. La pregunta entonces gira en torno a cómo se desarrolla una orientación a consumir, como lo que era inmoral se vuelve moral, deseado y perseguido. Las bases éticas del consumismo se vinculan con la idea de una falta de límites en la economía, que podría representarse en lo que Fromm nombra como la producción ilimitada. El rol del consumidor moderno es estar deseante bajo cualquier tiempo y circunstancia. Esto, nos dirá, -y se vuelve central- no tiene una base en la psicología humana sino en la cultura de una civilización.

Para Campbell, el consumidor no nació sino que fue producido por procesos que le han enseñado a querer cosas, a tener necesidades que se produjeron o inventaron socialmente. Es un sujeto en estado de descontento, de tensión económica y de satisfacción incompleta. Otro aspecto central de las llamadas bases éticas del consumo moderno tiene que ver no solo con querer desear cosas todo el tiempo sino también con hacerlo sin importar si se adquieren o consumen, como un estado deseante que no tiene que ver con una posesión efectiva.

El autor pone el ejemplo de alguien preguntando: ¿qué querés para tu cumpleaños o navidad? Ante la respuesta de "no quiero nada", lo que sigue es: "algo debes querer". Lo que dirá es que lo que caracteriza al consumidor moderno, no es querer algo como un fin en sí mismo sino querer sin fin. Que la experiencia de querer consumir sea el fin en sí mismo, no importa que. Esto lidera, inicia un proceso de necesidades o deseos producidos, armando un juego de tensión entre desear y encontrarse insatisfecho de modo constante.

Deseo de desear, búsqueda constante de experiencias, son aspectos centrales en este consumidor que se busca describir. En el consumo moderno hay una actitud positiva hacia "querer". Pero que yace sobre un sentido de la obligación. No es racional, calculador o simplemente auto-centrado, pero tampoco es descontrolado, irracional o impulsivo. En la esfera del consumo, la obligación individual es la de satisfacer los deseos y en la esfera de la producción la obligación es satisfacerlos a través del mercado.

El consumismo moderno es posible gracias a una serie de modificaciones tecnológicas, sociales y económicas. Entre estas modificaciones podemos nombrar: la producción masiva, la publicidad y la venta a crédito como elementos que lo hicieron posible. El asunto central, dirá Campbell (1983), es que incluso cuando se identifican todos estos elementos, el consumismo moderno sigue sin ser explicado en tanto patrón de comportamiento significativo para el análisis de lo social. En línea con intentar explicar dicho patrón de comportamiento, incorpora algunos elementos del romanticismo que podrían tener afinidad con la conducta de consumo moderno.

El Romanticismo, como movimiento se da en paralelo con la revolución industrial, en países como Inglaterra, Francia y Alemania principalmente, comenzando a fines del siglo XVII. Campbell alude a cierto consenso en considerar el romanticismo como una forma de ver la vida, de las creencias y los pensamientos. Taylor (2006), por su parte, describe algunos rasgos de la modernidad que van en la misma dirección y que se relaciona con un modo particular de concebir la individualidad, que atañe a otorgarle valor a la vida, al desarrollo de la personalidad, un gran énfasis puesto en alcanzar el bienestar humano, la evitación del sufrimiento y que le otorga a la actividad productiva y a la vida familiar un lugar central.

En primer lugar, el romanticismo se erige como crítica a la industrialización, por pensar este proceso como causa del mal que produce el materialismo, el utilitarismo y el racionalismo. En segundo lugar podemos identificar una concepción de las personas como sujetos únicos. Rousseau, puede ser una influencia en esta línea de pensamiento, al proponer la auto-expresión como condición para la felicidad. En su obra, la alusión al self empieza a tener presencia.

Este "movimiento" no solo afecto a algunos sectores de la sociedad, sino que puede considerarse como algo popular e incluso vulgar. Se popularizó y extendió gracias a que la literatura, "la novela moderna" (Taylor, 2006) y el modo en que esta se volvió accesible para gran número de personas gracias a la industrialización y los cambios en las formas de acceder a la misma. El relato de amor a través de la literatura retrata aquello que el autor describe como "la expansión del yo", al considerar las experiencias de enamoramiento como formas de la experiencia psicológica individual ${ }^{1}$. Constituyeron un retrato de lo particular, un relato sobre la vida de personas corrientes con gran nivel de detalle que exhibía una "nueva conciencia" (Taylor, 2006: 395).

Así, la alusión al carácter único de la personalidad se convirtió en una poderosa doctrina. El self se convirtió en una especie de dios a quien todos debemos obediencia. Para el romanticismo el hombre es un reservorio de posibilidades que pueden realizarse y concretarse solo si las fuerzas opresivas de la sociedad retroceden. El self es una fuerza plagada de sentimientos que la sociedad constriñe. Para el romanticismo, los sentimientos son una forma de conocimiento, el corazón es el locus de ideas innatas. El hombre puede conocer a través de la intuición, de sus instintos. Las sensaciones son un componente central de la experien-

1 Sobre este punto, ver Taylor (2006: 399), que al describir ciertos rasgos de la cultura de la modernidad, desarrolla el modo en que el matrimonio basado en el afecto comienza a ser idealizado, y dice: "la nueva comprensión del matrimonio naturalmente corre paralela a una nueva individualización e interiorización". 
cia, tanto física como moral, es la manera de llegar a una sabiduría más profunda. Romero (2006) agregará no solo el peso que tiene el realismo en este proceso, donde el hombre a partir de su experiencia es capaz de conocer el mundo sino que en tanto sujeto de percepción y sensaciones puede vivir grandes aventuras eróticas, creativas así como de ascenso económico.

Este conocer a través de la experiencia no solo enfatiza el valor de los sentimientos sino también el valor del placer. Este es para pensadores de la época el atributo de la vida. Este atributo no excluye el dolor o la tristeza, como emociones que pueden estar relacionadas con el placer también. El romanticismo -como ya se ha dicho- implica considerar el "uno mismo" o self como algo único y excepcional. El romántico, dice Campbell (1983) es quien se descubre a sí mismo como centro y tiene una concepción particular de la libertad, asociada con la realización personal.

La realización personal, el auto-desarrollo, la persecución del placer y la búsqueda de experiencias son elementos que para Campbell podrían ser afines a la orientación del consumo moderno. En dicho sentido, nos dirá que el consumo está relacionado con el yo y la afirmación de la identidad personal; y que, en tanto práctica, es visto como un modo o una vía para expresar tanto la identidad como la posición social.

Ambos autores nos permiten hacer algunas reflexiones sobre el nexo entre consumo y emociones. En ambos están presentes ciertos atributos de los sujetos, se habla de hedonismo, de la importancia que cobra en los procesos descritos el "uno mismo", la realización personal, el deseo, las experiencias, así como el modo en que perseguir el placer se "destraba", se vuelve algo no solo permitido, sino deseado. Los dos trabajos muestran modificaciones en las formas del sentir que podrían ser afines con las prácticas de uso/ adquisición de objetos. Todas estas formas terminan reflejando que si bien se alude a los conceptos de conducta o comportamiento, los cambios introducidos se relacionan con procesos sociales complejos -involucran varios aspectos y dimensiones- que no tienen base en la psicología individual. De este modo, los trabajos citados, son un aporte para problematizar el modo en que la proliferación de prácticas de consumo se vincula con transformaciones en los sujetos y sus emociones.

\section{El hombre de consumo del siglo XXI}

Hasta aquí, hemos recolectado análisis sobre la práctica de consumo y sobre el sujeto consumidor. A continuación recuperamos una perspectiva del siglo XXI que viene a delinear los rasgos del sujeto de consumo de la actualidad. A partir de allí intentaremos un diálogo entre dicha perspectiva y las que hemos descrito en el apartado anterior intentando decir algunas líneas más sobre la relación entre consumo y emociones sociales.

El capitalismo ha estado desde sus orígenes atado a las prácticas de consumo (Scribano, 2015; Trentmann, 2016), y como hemos señalado desde el comienzo también estuvo atado a diferentes emociones y formas de vivenciar el mundo. Estas formas han ido atravesando transformaciones -como también hemos visto-, hasta llegar a delinear el hombre de consumo del siglo XXI, que Scribano (2015) va a describir por medio de algunos elementos como la normalización y el disfrute inmediato. Este hombre del siglo XXI nos da una pista más, otra forma de seguir el modo en que las emociones se van configurando, en el habitar de los sujetos en el mundo y el modo en que históricamente esto se va transformando.

Así como el modo de producción capitalista ha estado atado al consumo, también ha intentado garantizar su reproducción. Este "hombre de consumo" se encuentra atravesado por las lógicas expropiatorias del capital, asociadas a los modos de extracción, producción y distribución vigentes, que cobran en los países del Sur Global la forma de múltiples y variadas expoliaciones y prácticas de dominación (Scribano, 2013). Las lógicas expropiatorias mencionadas podrían desagregarse en algunos elementos: la concentración monopólica, la extracción y apropiación de los recursos naturales ${ }^{2}$, así como la energía en todas sus variantes: ejemplos de esto podrían desde el petróleo hasta la energía corporal que es susceptible de ser apropiada y consumida (Scribano, 2013).

Las emociones de los sujetos -que parecieran ser algo íntimo e individual- son la condición de posibilidad de la reproducción del modo de acumulación actual ${ }^{3}$, por lo que su perspectiva intenta ahondar en los

\footnotetext{
2 Para un mayor desarrollo, ver: Scribano, A. (2013) La religión neo-colonial como la forma actual de la economía política de la moral. En: De Prácticas y discursos. Universidad Nacional del Nordeste. Centro de Estudios Sociales. Año 2, N`2.

3 Scribano (2013), alude a que para que esto sea posible son necesarios ciertos mecanismos de soportabilidad social y dispositivos de regulación de las sensaciones. Los citados mecanismos de soportabilidad social aluden a un conjunto de prácticas que operan casi desapercibidamente actuando desde la costumbre, el sentido común y las sensaciones, que parecen ser algo muy propio e íntimo del ser humano. La vida se presenta para los individuos como un "siempre asî", que combina la imposibilidad de hacer las cosas de otro modo con la naturalización de las faltas estructurales. Estos mecanismos se orientan a la evitación sistemática del conflicto que suponen las expropiaciones y depredaciones energéticas del capital (Scribano y Cervio; 2010). Los dispositivos de regulación de las sensaciones consisten en determinados procesos de selección, clasificación y elaboración de las percepciones socialmente determinadas y distribuidas que dan por resultado que los sujetos adopten una determinada manera de verse en el mundo en relación
} 
modos en que consumo y desigualdades son procesos que operan en simultáneo.

Lo que dirá Scribano sobre la desconexión, es que las distintas formas del sujeto, las posiciones que asume, como consumidor, como productor, ciudadano o espectador no logran estar conectadas. Lo que se hace en cada espacio de la vida está separado, de modo que no se llegan a percibir contradicciones o incompatibilidades. Por esto, sostiene que la normalización procura "un estado de flujo a-conflictivo" (2015: 46). El sujeto que aparece en las redes sociales, que va a trabajar, que compra algo, no aparecen como un sujeto único. Un ejemplo de esto podría ser el modo en que las redes sociales permiten exhibir un extracto de la vida, un momento de disfrute, que quizá no guarda gran relación con los demás aspectos de la vida de una persona.

El modo en que se produce esto es a través de la normalización. La misma se vincula con formas de estabilización, determinadas formas de relación entre las personas y la repetición compulsiva de actos de consumo. "La normalización del siglo XXI produce/reproduce una «separación» entre las acciones de los individuos de manera tal que se modifica la noción misma de inter-acción pero principalmente los entramados de las acciones de un mismo individuo entre sí, de una práctica con otra práctica performativa por el mismo individuo" (Scribano, 2015: 45), lo que alude a la fragmentación, como regla de los procesos identitarios (Scribano, 2005). Lo dicho, alude a formas de desconexión entre unas personas con otras y las personas consigo mismas. La estabilización, por su parte se vincula con un estado de evitación conflictual, con cierto manejo de la realidad en un registro esperable, sin sobresaltos.

El disfrute inmediato, por su parte, es un dispositivo de disminución de la ansiedad que implica la generación constante -repetición compulsiva- de objetos para la satisfacción a través del consumo. Scribano (2015) sostiene que hay una forma del sentir social -una sensibilidad- relacionada con la realización de esfuerzos constantes por seguir consumiendo a la vez que opera un imperativo que compele al sujeto al goce, a la felicidad y a la evitación del conflicto. No es más -ni menos- que "la vida como flujo, bajo la cobertura explicativa de pasarla bien" (Scribano, 2015: 58). Tiene que ver con aquel momento que le da sentido al acto de consumo y "borra" el recuerdo de los esfuerzos realizados. Baudrillard (2009) en ese sentido también dirá que el consumo apunta a esa "felicidad por defecto" que es la resolución de las tensiones.

La ligazón que resalta Scribano, entre el consumo y las desigualdades y expropiaciones, tienen relación con aquello que nombra Fromm al mencionar la estructura del carácter vinculada al tener con el fin de una ilusión. El consumo ya no se relaciona -desde sus propios practicantes- con el progreso sin límites. Es una práctica que prolifera más allá del nivel de ingresos, más allá de que el progreso no suceda y que las desigualdades se exacerben. Tiene que ver con prácticas producto de una transformación en la forma de sentir de los sujetos y de interactuar entre sí. Con sujetos desconectados, normalizados y estabilizados, que repiten prácticas de adquisición/ uso productoras de sensaciones de disfrute momentáneas que disminuyen el peso de las molestias cotidianas.

Lo propio del hombre del consumo del siglo XXI es esa desconexión en sintonía con el ser compelido al disfrute. La publicidad seduce hacia la compra al mismo tiempo que algunas políticas públicas la fomentan desde un ideario que liga consumo con producción y crecimiento. En ligazón con los demás trabajos que se han recuperado aquí, debemos recuperar que sin importar si existen o no esperanzas sobre el futuro, hay tanto en Fromm (2013), en Campbell (1983) como en Scribano (2015) una mención a la práctica de consumo como un fin en sí mismo, que trasciende el obtener o no objetos y/o servicios, tiene más que ver con un estado de deseo, de anhelo constante.

El asunto de problematizar el consumo y las emociones tiene que ver, no tanto con la adquisición concreta y creciente, sino con las modificaciones en la estructura del carácter que menciona Fromm, o las conductas del consumidor moderno que menciona Campbell. Es aquel querer sin fin, que para tener lugar requirió de transformaciones en los sujetos como las que se han intentado describir. La orientación al placer, o al disfrute se mantiene ligada a los actos de consumo. Lo que agregará Scribano (2015) al análisis -un aporte al problema en cuestión desde la óptica del siglo XXI-, es un estado de desconexión de unos sujetos con otros, la evitación del conflicto y la repetición compulsiva en un contexto de aplicación de políticas neo-keynesianas de incentivo y expansión del consumo ${ }^{4}$. Si en el momento que Fromm escribe ya se avizoraba "el fin de la ilusión" asociada al progreso y felicidad sin límites, la fotografía del siglo XXI que propone Scribano, nos permite un cuestionamiento en dos direcciones: en primer lugar seguir preguntán-

con su contexto de clase.

4 "El eje de las políticas económicas de muchos de los estados del Sur Global es su carácter neo-keynesiano, por lo cual los incentivos y gestión de la expansión del consumo se transforman en una de sus principales herramientas. Créditos para el consumo, subsidios para el consumo, incentivos 'oficiales' para el consumo se cruzan y superponen con el estado consolidado y en continuo desarrollo del capitalismo en su contradicción depredación/consumo. Se producen/reproducen así unas sociedades estructuradas en torno a un conjunto de sensibilidades cuyo contexto de elaboración lo constituye los continuos esfuerzos por seguir consumiendo" (Scribano, 2015: 43). 
donos por las emociones que históricamente se asocian a las prácticas de consumo -siempre cambiantes- y en segundo lugar visualizar los procesos de desigualdad que no imposibilitan ni hacen obstáculo alguno a la proliferación creciente de prácticas de adquisición/uso.

\section{A modo de cierre momentáneo}

El objetivo de este escrito fue reflexionar sobre el modo en que las emociones se relacionan con el desarrollo de cierta orientación a consumir, preguntarnos acerca de cómo lo que en un momento histórico fue considerado inmoral se fue volviendo aceptable e incluso deseado. En este sentido, hemos intentado revisar las formas en que procesos, sucesos y/o transformaciones perfilaron la persecución del placer, y de la satisfacción auto-centrada como una forma de vida.

Esta forma de vida, fue consolidándose muy lentamente, por lo que no podemos pensarla como algo propio de la actualidad. Lo que desarrolla Fromm como las alusiones al placer desde el terreno de la filosofía así como las ilusiones que conllevó el proceso industrial fueron contribuyendo a la conformación de unas emociones que persiguen de modo creciente actos de consumo o que se habilitan un estado de "querer sin fin", como ya hemos dicho a lo largo de este trabajo. Campbell (1983), por su parte, describiendo rasgos del romanticismo intenta aportar sobre el modo en que el self, ganó importancia y se convirtió en el centro de las prácticas de consumo. De hecho, la centralización del "yo", o el desarrollo de la individualidad (Taylor, 2006) parecen ser un punto que, aquellos que estamos interesados en hacer estudios sobre la práctica de consumo debemos -al menos- transitar.

Ahora bien, haciendo un pasaje hacia la actualidad retomamos los aportes de Scribano $(2013 ; 2015)$, donde el consumo se presenta como una práctica que apunta a la consecución de un disfrute aquí y ahora. Recuperando un contexto de grandes desigualdades a nivel global, el autor nos plantea como cierta regulación de las sensaciones hace posible que los sujetos vivan en el mundo compelidos a la compra y uso, en un estado de desconexión de unos con otros y con ciertos aspectos de la realidad que habitan. Lo que describe, son transformaciones en las formas de interacción de los sujetos y con los modos en que estos viven en un contexto de desigualdades y expropiaciones atravesado por las prácticas de consumo como vías para disolver tensiones.

Si bien no es un aspecto muy desarrollado en el cuerpo de este escrito, el análisis de esta sociabilidad de consumo, debe tener presente su colaboración con la disolución de las tensiones y el divorcio -siempre conflictivo- entre el lugar de consumidor y el lugar de trabajador, que fue propio de mediados de siglo XX. Podríamos comenzar a considerar que la particularidad actual, quizá resida en que " «Comprar cosas» no es la consecuencia o reflejo de un lugar ocupado en el proceso productivo, como en los modelos de producción y consumo fordista. El consumo se ha vuelto preeminente, conformando modos de sociabilidad, que implican no solo un anhelo constante de objetos sino un modo de relación con el mundo, en el sentido que le da Fromm (2013) al realizar una diferenciación entre el ser y el tener como modos fundamentales de la experiencia humana.

\section{Bibliografía}

Alonso, P. (2005) La era del consumo. Barcelona: Siglo XXI.

Bataille, G. (1933) La noción de gasto. La critique Sociale, $N^{\circ} 7$. Recuperado de: http: //economiainternacional.sociales.uba.ar/files/2011/03/Bataille_La-nocion-de-gasto.pdf

Baudrillard, J. (2010) El sistema de los objetos. México: Siglo XXI editores.

Bauman, Z. (2007) Vida de consumo. Buenos Aires: Fondo de Cultura Económica

Bericat Alastuey, E. (2012). Emociones. Sociopedia.isa, pp. 1-13.

Campbell, C. (1983) Romanticism and The Consumer Ethic: Intimations of a Weber-Style Thesis. Sociological Analysis, Vol. 44, No. 4 (Winter, 1983), pp. 279-295. Published by: Oxford University Press.

Dettano, A., Lava, M. (2014) Entablando vinculaciones entre la sociedad de consumo, las sensibilidades y las políticas sociales desde el sur global. Boletín Científico Sapiens Research Vol. 4(2)-2014. pp: 27-32. Disponible en: http: //www.sapiensresearch.org/images/pdf/v4n2/V4N2_Sociologando_1. pdf

Douglas, M.; Isherwood, B. (1990) El mundo de los bienes: hacia una antropología del consumo. México: Grijalbo.

Elias, N. (2009) El proceso de la civilización. México: Fondo de Cultura Económica.

Fava, J. (2009) Prefacio. La parte Maldita y apuntes Inéditos. Buenos Aires: Las Cuarenta.

Fromm, E. (2013) ¿Tener o ser? Buenos Aires: Fondo de Cultura Económica. 
Hochschild A. (2011) La mercantilización de la vida íntima. Apuntes de la casa y el trabajo. Buenos Aires: Ed. Katz.

Jameson, F. (2002) El giro cultural. Buenos Aires: Manantial.

Lipovetsky, G. (2007) La felicidad Paradójica. Barcelona: Editorial Anagrama.

Marx, K. (2008) El Capital (Tomo I, Vol.1). Buenos Aires: Siglo Veintiuno editores.

Romero, J. L. (2006) Estudio de la mentalidad burguesa. Buenos Aires: Alianza Editorial.

Sánchez Aguirre, R. (2013) Apuntes sobre la construcción conceptual de las emociones y los cuerpos. Revista Latinoamericana de Estudios sobre Cuerpos, Emociones y Sociedad. Relaces, №13. Año 5. Diciembre 2013, Marzo 2014. pp. 75-86. Recuperado de: http: //www.relaces.com.ar/index.php/ relaces/article/view/252

Scribano, A. (2005) Itinerarios de la protesta y del conflicto social. Córdoba: Centro de Estudios Avanzados, Universidad Nacional de Córdoba.

Scribano, A. (2009) A modo de epílogo. ¿Por qué una mirada sociológica de los cuerpos y las emociones?, en Figari, C. y Scribano, A. (Comp.), Cuerpo(s), Subjetividad(es) y Conflicto(s). Hacia una sociología de los cuerpos y las emociones desde Latinoamérica. (pp.141-151). Buenos Aires: CICCUS, CLACSO.

Scribano, A.; Cervio, A. (2010) La ciudad neocolonial: Ausencias, Síntomas y mensajes del poder en la Argentina del Siglo XXI. En Sociólogica N2.

Scribano, A. (2013) La religión neo-colonial como la forma actual de la economía política de la moral. De Prácticas y discursos, $\mathrm{N}^{\circ} 2$, Año 2. Universidad Nacional del Nordeste. Centro de Estudios Sociales.

Scribano, A. (2015) ¡Disfrútalo! Una aproximación a la economía política de la moral desde el consumo. Buenos Aires: El Aleph.

Taylor, C. (2006) Fuentes del yo. La construcción de la identidad moderna. España: Editorial Paidós.

Trentmann, F. (2016) The empire of things. How we became a world of consumers, from the fifteenth Century to the twenty-first. United Kingdom: Penguin random house.

Veblen, T. (1944) La teoría de la clase ociosa. México: Fondo de cultura económica. 